\title{
关于 $\frac{1}{0} 、 \frac{0}{0}$ 和 $0^{\circ}$ 的讨论及完全定理： $\frac{1}{0}-\frac{1}{0}=\frac{0}{0}-0^{\circ}$
}

Discussion on $\frac{1}{0} 、 \frac{0}{0}$ and $0^{0}$ and Complete Theorem: $\frac{1}{0}-\frac{1}{0}=\frac{0}{0}-0^{0}$

曹为文

Weiwen Cao

银川英才学校 中国·宁夏 银川 750002

Yinchuan Yingcai School, Yinchuan, Ningxia, 750002, China

摘 要: 0 不能当作分母, 这不仅导致 $\frac{1}{0} 、 \frac{0}{0}$ 和 $0^{0}$ 没有意义, 而且也使许多函数被迫出现各种各样的间断点, 现有的许 多公式也会因此受到诸多限制。更为重要的是，人们对于数学的研究也在一定程度和方向上受到某些束缚。因此赋予 0 做 分母正当的权利, 进而完善现行的运算法则, 对于解决一系列的、长期困扰数学领域的问题, 填补许多一直被人们忽视的 研究空白, 并将数学研究推向更高层次具有重要的意义。

Abstract: 0 can' t being the denominator not only makes $\frac{1}{0} 、 \frac{0}{0}$ and $0^{0}$ meaningless, but also forces many functions to have a variety of discontinuities, limiting many existing formulas. What' s more, people' $s$ research on mathematics is also bound up to some extent and direction. Therefore, it is of great significance to endue 0 the denominator and improve the existing algorithm, to solve a series of problems that have long troubled the field of mathematics, to fill in many research gaps that have been ignored, and to promote the research of mathematics to a higher level.

关键词: $0 ; \frac{1}{0} ; \frac{0}{0} ; 0^{0}$; 极数; 完全定理

Keywords : $0 ; \frac{1}{0} ; \frac{0}{0} ; 0^{0}$; number of poles; completeness theorem

DOI : $10.36012 /$ sde.v2i11.2397

\section{1 引言}

长期以来人们都坚持认为, 0 没有倒数, 0 也不能当做 除数。更有甚者, 0 作为底数或者指数也要受到严格的限制,

即 $\frac{1}{0} 、 \frac{0}{0}$ 和 $0^{0}$ 均无意义。否定的理由似乎也很充分 : 第一， 如果 0 做除数, 被除数则分为 0 和非 0 两种情况。(1)如果被 除数是 0 , 那么无论商为何数, 商与除数 0 的积都为 0 , 所 以商就有无数种可能，即商不确定；(2)如果被除数非 0 , 那 么无论商为何数，商与除数 0 的积都为 0 , 但是此时被除数 非 0 , 从而也不可能。第二,一个正整数 $m$ (被除数) 除以 另一个正整数 $n$ (除数), $\frac{m}{n}$ 意为将被除数 $m$ 平均分成 $n$ 份 以后每一份的大小。所以当 $n=0$ 时, $\frac{m}{0}$ 的意义就是要把一 个被除数平均分成 0 份，这当然是没有意义的。第三，在爱 因斯坦的相对论当中，物体的质量 $m$ 和速度 $v$ 存在着特定
的关系: $m=\frac{m_{0}}{\sqrt{1-\left(\frac{v}{c}\right)^{2}}}$ 。即物体的速度 $v$ 越快, 它的质量 $m$ 就会越大。当速度 $\mathrm{v}$ 无限趋近于光速 $c$ 的时候，公式的分 母 $\sqrt{1-\left(\frac{v}{c}\right)^{2}}$ 就会无限趋近于 0 , 那么物体的质量 $m$ 就会趋 近于 $\infty$ 。一旦物体达到光速, 即 $v=c$ 时, $\sqrt{1-\left(\frac{v}{c}\right)^{2}}=0$, 那 么物体的质量 $m=\frac{m_{0}}{0}$ ，这显然有悖常理。换句话说，静态 质量 $m_{0} \neq 0$ 的物体是无法达到光速的。所以构成光的“光 子”，在相对论中的静态质量 $m_{0}=0$ 。 即只有静态质量为 0 的 物体，才能够达到光速。综上所述，0不能做除数。

虽然上述理由看似非常充分，但也并非无解可击。对 于第一条理由, 当按运算法则运算, 结果存在多种可能的时 候, 可以强行规定其中一种作为最终结果。例如， $\overrightarrow{0}$ 因为长 度为 0 , 所以它的方向是任意的。这就意味着 $\overrightarrow{0}$ 与任一非零

【作者简介】曹为文 (1988 ), 男, 山东济南人, 从事数学教学研究工作。 
向量的夹角也可以是任意的，但是规定 $\overrightarrow{0}$ 与任一非零向量 平行 ${ }^{[1]}$ 。对于第二条理由, 它本身并不算是一个怎么高明的 “由头”。如果第二条理由成立, 那么诸如 $\frac{-1}{-2} 、 \frac{-3}{4} 、 \frac{5}{-6}$ 、 $\frac{7}{8.9} 、 \frac{\pi}{10} 、 \frac{0}{e}$, 以及 $i^{2}=1$ 等均无意义。至于第三条理由， 虽然看似高深莫测，实际却是自相矛盾。既然光子的速度满 足 $v=c$, 而且相对论也认为光子的静态质量 $m_{0}=0$, 难道这 不是在默认质增公式的分母 $\sqrt{1-\left(\frac{v}{c}\right)^{2}}$ 可以为 0 , 而且 $\frac{0}{0}$ 也 是存在的吗? 总而言之, 所谓的 0 不能做除数的三大理由看 似充分, 一旦咬文嚼字地细究起来, 没有任何一个经得起推 敲。然而正是在这些传统、偏狭、晦涩的观点的误导之下， 人们自始至终地坚持认为 0 不能做除数, 从而也就认为 $\frac{0}{0}$ 没有意义。又因为 $0^{0}=0^{1-1}=0^{1} \times 0^{-1}=0 \times \frac{1}{0}=\frac{0}{0}$, 所以 $0^{0}$ 也没有意义。这就导致 $y=\tan x 、 y=\frac{1}{x} 、 y=x^{0} 、 y=0^{x}$ 及同类 型的函数必然出现若干间断点。如果赋予 $\frac{1}{0} 、 \frac{0}{0}$ 和 $0^{0}$ 正当 的含义，并且改进运算法则，那么不仅可以去除上述间断点， 也会使得运算法则更加完善。

已知 $C_{n}^{0}=C_{n}^{n}=1$, 那么 $\mathrm{c}_{0}{ }^{0}$ 又是多少呢? 因 为 $C_{n}^{m}=\frac{n !}{m !(n-m) !}$, 所 以当 $n>0, m=0$ 时, 即 有 $C_{n}^{0}=\frac{n !}{0 !(n-0) !}=\frac{n !}{0 ! \cdot n !}=\frac{1}{0 !}=1$, 所以 $0 !=1$ 。这是 一个 重要的结论, 因为众所周知阶乘公式 $n !=n \times(n-1) \times$ $(n-2) \times \cdots \times 3 \times 2 \times 1$ 并不适合 0 ! 的运算。根据上式, $C_{0}^{0}=\frac{0 !}{0 ! \times(0-0) !}=\frac{0 !}{0 ! \times 0 !}=\frac{1}{1 \times 1}=\frac{1}{1}=1$, 所以 $C_{0}^{0}=1$ 。实际 上这是一个远比 $0 !=1$ 更为重要的结论, 因为 $C_{0}^{0}=1$ 恰恰是 对二项式定理的重要补充!

根据二项式定理 $:(a+b)^{n}=C_{n}^{0} a^{n}+C_{n}^{1} a^{n-1} b^{1}+\cdots+C_{n}^{k} a^{n-k} b^{k}$ $+\cdots+C_{n}^{n} b^{n}$ 。当 $n=0, a=1, b=1$ 时, 则有 $(a-b)^{n}=(1-1)^{\circ}$ $=\mathrm{c}_{0}{ }^{0}(1)^{0}(-1)^{0}=1 \times 1 \times 1=1=0^{0}$, 由此可知 $0^{0}=1$ 。又因 为 $0^{0}=\frac{0}{0}$ (参见上文), 所以 $\frac{1}{0} 、 \frac{0}{0}$ 和 $0^{0}$ 必然存在, 且有 意义。当然, 也可以根据等比性质得到相同的结论 : 如果 $\frac{a}{b}=\frac{c}{d}=\frac{e}{f}=\cdots=\frac{m}{n}$ 那么 $\frac{a+c+e+\cdots+m}{b+d+f+\cdots+n}=\frac{a}{b}$ 。由此可知， 如果 $\frac{1}{1}=\frac{-1}{-1}$, 那么 $\frac{1-1}{1-1}=\frac{0}{0}=\frac{1}{1}=\frac{-1}{-1}=1$, 所以 $\frac{0}{0}=1$ 。
如果 $\frac{1}{0}$ 存在, 那么它的含义是什么? 对于反比例函数 $y=\frac{1}{x}$, 当 $x \rightarrow 0$ 时, $\lim _{x \rightarrow 0^{+}} \frac{1}{x}=+\infty, \lim _{x \rightarrow 0^{-}} \frac{1}{x}=-\infty$ 。可延续这种 思路追问下去就会提出这样一个问题, 当 $x=0$ 时, $y=\frac{1}{x}$ 的 值又是什么呢? 对于这个问题, 一般认为, $x$ 只能无限趋近 于 0 ，但是永远不能到达 ${ }^{[2]}$ 。这使人不禁感到一种既“可望 而不可及” 又莫名其妙的愤愤不平：既然 $x$ 可以轻易地穿梭 整个数轴, 到达任何一个数值, 为什么就是不能到达 0 呢? 这就好比人们制造了一艘风驰电掣的宇宙飞船，它可以刹那 之间轻松自如地穿梭整个宇宙, 到达任何一个它想探索的 天体。但是它却无法开进发射自己的机舱，只能永远无限 趋近一样。因此人们理所应当赋予 0 做除数的权利, 使得 分母可以为 0 , 并且赋予 $\frac{1}{0}$ “极数”—“最大的数” 的含 义。即 $\frac{1}{0}$ 是人们所知的数的终极, 再也没有比 $\frac{1}{0}$ 更大的数 了。为了表述 $\frac{1}{0} 、 \frac{0}{0}$ 和 $0^{0}$ 的数值及其关系，使用如下等式 : $\frac{1}{0}-\frac{1}{0}=\frac{0}{0}-0^{0}$, 并将这个等式及其相关内容一起称为“完 全定理”。引入“完全定理” 以后, 再来研究如下几个重要 的问题。

2 如何通过 “极数” 概念重新认识点、线、面、 体及其相互关系

已知线由无数个点所构成, 面由无数条线所构成, 体 由无数个面所构成。然而这种说法也存在着一些问题, 例 如，直线上的任意两点之间总会存在一定的间距 $\sigma$ ，当然 可以利用二分法不断地缩小这个间距: 当分割 $n$ 次以后, 两 点之间的间距变为 $\frac{\sigma}{2^{n}}$ 。然而无论 $n$ 取何值, 即便 $n \rightarrow \infty$, 总 有 $\frac{\sigma}{2^{+\infty}}>0$, 这意味着直线并非连续不断, 而是存在无数个 间断点。恰如《庄子·天下》所言! 一尺之捶, 日取其半, 万世不竭”。另一方面, 既然点没有直径、面积和体积, 那 么线自然也就不应该存在长度、面积和体积了。以此类推， 面和体也不应该存在面积和体积, 难道这不是一个悖论吗? 尽管在实际的学习、教育和研究当中，经常在纸面或者黑板 上通过“累积”点、线、面形成长度、面积和体积的概念, 但这其实是因为人们使用工具绘制的点、线、面的确具有一 定的直径、面积和体积所致。这个道理放在物理学中同样适 用，甚至恰为佐证一倘若原子没有直径、面积和体积，物 
体自然也就没有长度、面积和体积了。可惜的是, 数学当中 所称的点确实没有直径、面积和体积 (见图 1)。

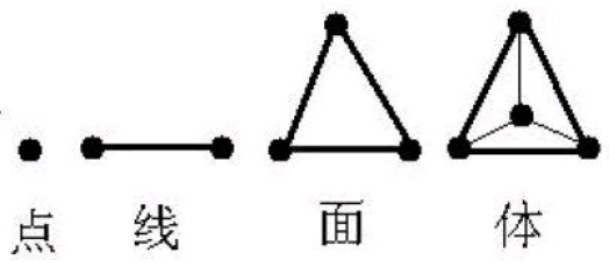

图 1 点、线、面、体

对于一段长为 1 的“线段”，它至多会有 $\frac{1}{0}$ 个点, 其 上相邻两点之间的间距： $\sigma=\frac{1}{\frac{1}{0}}=1 \times 0=0$, 该段“线段” 的 面积 $s=0 \times \frac{1}{0}=1$, 体积 $v=0 \times \frac{1}{0}=1$ 。那么边长为 1 的 正方形的面积: $S=1 \times 1=1$, 边长为 1 的立方体的体积： $\mathrm{V}=1 \times 1 \times 1=1$ 。对于长为 $l(l \geq 1)$ 的“线段”，它的面积和 体积在数值上也各为 $l$, 此时“线段” 上至多会有 $l \cdot \frac{1}{0}=\frac{1}{0}$ 个点。当 $0 \leq k<1$ 时, 它的面积和体积在数值上均为 $l$, 此时 “线段”上至多会有 $l \cdot \frac{1}{0}=\frac{l}{0}$ (不能约去 $l$, 参见后文“极数” 的运算法则) 个点。如此一来, 便为后续线、面和体的长度、 面积和体积的“累积”做好了铺垫。不过也会随之得到一个 令人诧异的结论：线 (包括射线) 最多可以达到 $\frac{1}{0}$ 的长度， 此时直线上也至多会有 $\frac{1}{0}$ 个点，那么相邻两点之间的间距： $\sigma=\frac{\frac{1}{0}}{\frac{1}{0}}=1$ 。这就意味着, 通常所说的直线上有无数个点, 实 际上是在研究直线的时候，将直线的一个有限的部分当做了 一条“线段”而已。不过由此也会发现，关于 $\frac{1}{0}$ 的运算具 有不可逆性。例如, 不可以说一个面积为 10 的图形具有 $\frac{10}{0}$ 个点, 它的确只有 $\frac{1}{0}$ 个点, 但是面积并非 $S=0 \times \frac{1}{0}=1$, 而 是 $S=10 \times 1=10$ 。

\section{3 引入“ 极数” 概念以后，如何重新认识间 断点}

对于 $y=\tan x 、 y=\frac{1}{x} 、 y=x^{0} 、 y=0^{x}$ 及同类型的函数，假 设它们的函数解析式为 $y=f(x)$, 并且通常会在它们的图像 上画出若干间断点。而一般又把间断点分成两类: 如果 $x_{0}$ 是函数 $f(x)$ 的间断点, 但左极限 $f\left(x^{*}\right)$ 及右极限 $f\left(x^{+}\right)$ 都存在, 那么 $x_{0}$ 称为函数 $f(x)$ 的第一类间断点。不是第
一类间断点的任何间断点，都称为第二类间断点。在第一类 间断点中，左右极限相等者称为可去间断点，不相等者称为跳 跃间断点。无穷间断点和振荡间断点显然是第二类间断点。

假设其中的某个间断点是 $x_{0}$, 并且在 $x_{0}$ 的某一邻域内还 有定义, 即 $\lim _{x \rightarrow x_{0}} f(x)=y_{0}$ 。现在给函数 $y=f(x)$ 赋值 $y_{0}$, 那 么必然存在 $\lim _{\Delta x \rightarrow 0} \Delta y=\lim _{\Delta x \rightarrow 0}\left[f\left(x_{0}+\Delta x\right)-f\left(x_{0}\right)\right]=0$ 。由此 可知, $y=f(x)$ 在 $x=x_{0}$ 处连续。以此类推，其他间断点的 问题也会随之迎刃而解。例如, 函数 $\mathrm{y}=\frac{x^{2}-1}{x-1}$ 在 $x=1$ 处 没有定义, 所以函数在 $x=1$ 处不连续。但当 $x$ 不断趋近 于 1 时, 函数在 $x=1$ 的左右两侧的极限存在并且相等, 即 $\lim _{x \rightarrow 1} \frac{x^{2}-1}{x-1}=\lim _{x \rightarrow 1}(x+1)=2$ (见图 2)。如果补充定义 : 令 $x=1$ 时， $y=2$, 则所给函数在 $x=1$ 处连续, 所以 $x=1$ 称为该函数的可 去间断点。

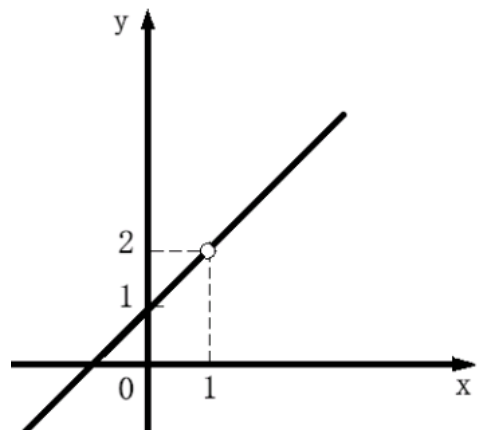

图 2

\section{4 既然 0 可以做分母，那么可以将公式中的} 分母直接替换为 0 吗

虽然赋予了 $\frac{1}{0}$ 正当的含义，但这并不意味着可以将公 式中的分母直接替换为 0 。这是因为, 要么未必能够按照完 善以后的运算法则得到这个分母为 0 的 公式”, 要么这些 公 式” 将会变得毫无意义, 下面举出几个例子予以说明：

例 1 , 对于截距式方程: $\frac{x}{a}+\frac{y}{b}=1$, 当 $a=b=0$ 的时 候, 直线通过原点。如果将分母直接替换为 0 , 就会得到: $\frac{x}{0}+\frac{y}{0}=1$ 。然而 对于一条通过原点的直线 (为了方便讨论, 假设 $k \geq 1), y=k x \Rightarrow \frac{y}{0}=\frac{k x}{0} \Rightarrow \frac{k x}{0}-\frac{y}{0}=0 \Rightarrow \frac{x}{0}-\frac{y}{0}=0$, 与 $\frac{x}{0}+\frac{y}{0}=1$ 形式并不相同。当直线为 $y$ 轴时 斜率 $k=\frac{1}{0}$ (参 见下文)。如果将其代入 $y=k x$ 中就会得到 $: y=\frac{1}{0} \cdot x$ 。又因 
此时 $x=0$, 于是便有: $y=\frac{1}{0} \times 0=1$, 显然这是错误的。因 为此时 $y$ 并非 $x$ 的函数: $x=0$ 时对应无数个 $y$ 值, 所以函数 解析式应为: $x=0$ 。

例 2 , 对于公差公式: $d=\frac{a_{n}-a_{m}}{n-m}$, 当 $m=n$ 时, $d=\frac{a_{n}-a_{m}}{n-m}=\frac{a_{n}-a_{n}}{n-n}=\frac{0}{0}=1$, 显然这是错的。因为一 个数和它本身不会存在“公差”的问题, 公差至少需要 两个数。当然也可以承认单独一个数拥有公差, 但是毫 无疑问这个公差只能是 0 , 那么问题出在哪里呢? 知道 $\left\{\begin{array}{l}a_{n}=a_{1}+(n-1) d \\ a_{m}=a_{1}+(m-1) d\end{array} \Rightarrow a_{n}-a_{m}=0+(n-m) d \Rightarrow a_{n}-a_{m}-0=(n-m) d\right.$ $\Rightarrow \frac{a_{n}-a_{m}}{n-m}-\frac{0}{n-m}=d \Rightarrow \frac{a_{n}-a_{n}}{n-n}-\frac{0}{n-n}=d \Rightarrow \frac{0}{0}-\frac{0}{0}=d \Rightarrow 1-1=d^{\prime}$ $\Rightarrow d=0$ 。值得注意的是, 倘若 $a_{n}-a_{m}-0=(n-m) d$ $\Rightarrow \frac{a_{n}-a_{m}-0}{n-m}=d \Rightarrow \frac{a_{n}-a_{n}-0}{n-n}=d \Rightarrow \frac{0-0}{0}=d \Rightarrow \frac{0}{0}=d$ $\Rightarrow d=1$, 结果显然是错的。这是因为 $\frac{0}{0}-\frac{0}{0}=1-1=0$, 但 是 $\frac{0-0}{0}=\frac{0}{0}=1$, 即 $\frac{0}{0}-\frac{0}{0} \neq \frac{0-0}{0}$ 。也就是说, $\frac{0-0}{0}$ 相对 $\frac{0}{0}-\frac{0}{0}$ 而言，实际上“遮蔽”了一次 $\frac{0}{0}$ 的运算。

例 3 , 对于正弦定理 : $\frac{a}{\sin A}=\frac{b}{\sin B}=\frac{c}{\sin C}=2 R, A$ 、 $B 、 C$ 分别代表 $\triangle A B C$ 的三个角度。其中任何一个角度为 0 都会使得正弦定理失去意义, 更不要说其他数据也会随之发 生变化了。

例 4 , 对于点到直线的距离公式: $d=\frac{|A x+B y+C|}{\sqrt{A^{2}+B^{2}}}$, 如果 $A=B=0$, 那么 $C=0$, 则直线方程没有意义, 也就不会 存在点到直线的距离了。

5 对于对数函数 $y=\log _{a} x ，(a>0)$, 当 $x \leq 0$ 时, 函数是否意义

对于对数函数 $y=\log _{\alpha} x,(a>0)$, 实际上 $x$ 可以任意取值。 当 $x<0$ 时, $y=\log _{a} x=\log _{a}(-x) \cdot(-1)=\log _{a}(-x) \cdot \mathrm{i}^{2}=\log _{a}$ $(-x)+\log _{a} i^{2}=\log _{a}(-x)+2 \log _{a} \mathrm{i}$ 。 当 $x=0$ 时, $y=\log _{\alpha} x=\log _{a} 0$ 。 如图 3 所示, 当 $a>1$ 时, $y=\log _{a} 0=-\frac{1}{0}$ 。当 $0<a<1$ 时, $y=\log _{a} 0=\frac{1}{0}$ 。当 $a=1$ 时, $y=\log _{a} 0$ 不存在。

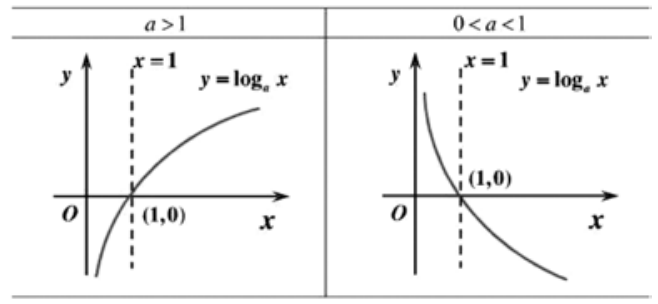

图 3

6 如何看待在爱因斯坦的相对论当中, 光子 的静态质量 $m_{0}=0$

对于质增方程 $m=\frac{m_{0}}{\sqrt{1-\left(\frac{v}{c}\right)^{2}}}$, 当 $m_{0} \neq 0$ 时, 物体的运 动速度 $v$ 越快, 动态质量 $m$ 越大 (见图 4)。当 $v=c$ 时， $m=\frac{m_{0}}{\sqrt{1-\left(\frac{v}{c}\right)^{2}}}=\frac{m_{0}}{\sqrt{1-\left(\frac{c}{c}\right)^{2}}}=\frac{m_{0}}{\sqrt{1-1^{2}}}=\frac{m_{0}}{\sqrt{1-1}}=\frac{m_{0}}{0}$, 显然这 是不可思议的。且不要说一个正常的物体, 即便质量惊人的 黑洞也不可能满足 $m=\frac{m_{0}}{0}$ 。但是对于光子而言, 它的速度 的确满足 $v=c$, 难道光子运动的时候没有质量吗? 当然不是, 如前所述, 只有光子的静态质量 $m_{0}=0$, 才会存在光子的动 态质量 : $m=\frac{m_{0}}{0}=\frac{0}{0}=1$ 。

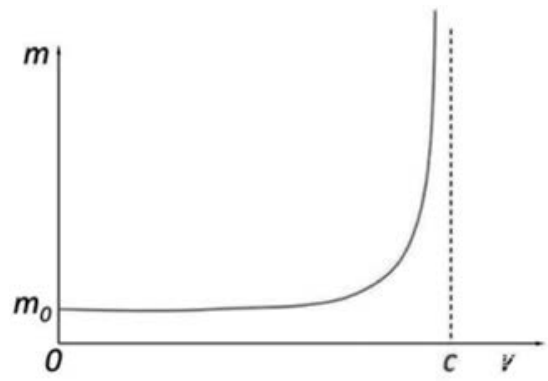

图 4

$7 \overrightarrow{0}$ 的方向是任意的, 但是规定 $\overrightarrow{0}$ 与任一非 零向量平行, 这种规定是否合理; 如果合理, 它们之间的夹角到底是 0 还是 $\pi, \overrightarrow{0}$ 与 $\overrightarrow{0}$ 之间 的位置关系如何

知道 $\cos \theta=\frac{\vec{a} \vec{b}}{|\vec{a}| \vec{b} \mid}=\frac{x_{1} x_{2}+y_{1} y_{2}}{\sqrt{x_{1}^{2}+y_{1}^{2}} \sqrt{x_{2}^{2}+y_{2}^{2}}}$, 其中 $\vec{a}=\left(x_{1}, y_{1}\right)$, $\vec{b}=\left(x_{2}, y_{2}\right)$ (见图 5)。假设 $\vec{a} \neq \overrightarrow{0}, \vec{b}=\overrightarrow{0}$, 即可得到： $\cos \theta=\frac{x_{1} \cdot 0+y_{1} \cdot 0}{\sqrt{x_{1}^{2}+y_{1}^{2}} \cdot \sqrt{0^{2}+0^{2}}}=\frac{0+0}{\sqrt{x_{1}^{2}+y_{1}^{2}} \times \sqrt{2} \times 0}=\frac{0}{\sqrt{x_{1}^{2}+y_{1}^{2}} \times 0}=\frac{0}{0}=1$, 所以 $\theta=0$ 。 可见 $\overrightarrow{0}$ 与任 一非零向量不但平 
行, 而且夹角为 0 。假设 $\vec{a}=\vec{b}=\overrightarrow{0}$, 即可得到： $\cos \theta=\frac{x_{1} \cdot 0+y_{1} \cdot 0}{\sqrt{x_{1}^{2}+y_{1}^{2}} \cdot \sqrt{0^{2}+0^{2}}}=\frac{0+0}{\sqrt{x_{1}^{2}+y_{1}^{2}} \times \sqrt{2} \times 0}=\frac{0}{\sqrt{x_{1}^{2}+y_{1}^{2}} \times 0}=\frac{0}{0}=1$. 所以 $\theta=0$ 。可见 $\overrightarrow{0}$ 与 $\overrightarrow{0}$ 不仅平行, 而且夹角为 0 。

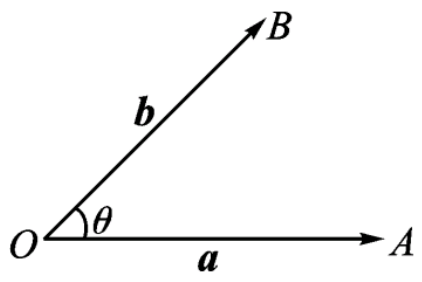

图 5

8 在公比 $q=1$ 的情况下，等比数列求和公式 $S_{n}=\frac{a_{1}\left(1-q^{n}\right)}{1-q}$ 是否适用

当 $q=1$ 时，等比数列求和公式 $S_{n}=\frac{a_{1}\left(1-q^{n}\right)}{1-q}$ 依然 适用，甚至可以将其推广至以 0 为项的常数列。首先 研 $a_{n} \neq 0$ 究, $q=1$ 的情况, 根据 $S_{n}=\frac{a_{1}\left(1-q^{n}\right)}{1-q}$ 可知 : $S_{1}=\frac{a_{1}\left(1-q^{1}\right)}{1-q}=\frac{a_{1}(1-q)}{1-q}=a_{1} ;$

$S_{2}=\frac{a_{1}\left(1-q^{2}\right)}{1-q}=\frac{a_{1}(1-q)(1+q)}{1-q}=a_{1}(1+q)=a_{1}(1+1)=2 a_{1}$; $S_{3}=\frac{a_{1}\left(1-q^{3}\right)}{1-q}=\frac{a_{1}(1-q)\left(1^{2}+1 \cdot q+q^{2}\right)}{1-q}=a_{1}\left(1^{2}+1 \cdot q+q^{2}\right)$$$
=a_{1}\left(1^{2}+1 \times 1+1^{2}\right)=3 a_{1}
$$

$S_{4}=\frac{a_{1}\left(1-q^{4}\right)}{1-q}=\frac{a_{1}\left(1-q^{2}\right)\left(1+q^{2}\right)}{1-q}=\frac{a_{1}(1-q)(1+q)\left(1+q^{2}\right)}{1-q}$

$=a_{1}(1+q)\left(1+q^{2}\right)=a_{1}(1+1)\left(1+1^{2}\right)=a_{1}(1+1)(1+1)$

$=a_{1} \times 2 \times 2=4 a_{1}$

$S_{5}=\frac{a_{1}\left(1-q^{5}\right)}{1-q}=\frac{a_{1}(1-q)\left(1^{4}+1^{3} \cdot q+1^{2} \cdot q^{2}+1 \cdot q^{3}+q^{4}\right)}{1-q}$

$=a_{1}\left(1^{4}+1^{3} \cdot q+1^{2} \cdot q^{2}+1 \cdot q^{3}+q^{4}\right)$

$=a_{1}\left(1^{4}+1^{3} \times 1+1^{2} \times 1^{2}+1 \times 1^{3}+1^{4}\right)=a_{1}(1+1+1+1+1)=5 a_{1}$

$S_{6}=\frac{a_{1}\left(1-q^{6}\right)}{1-q}=\frac{a_{1}\left(1-q^{3}\right)\left(1+q^{3}\right)}{1-q}=\frac{a_{1}(1-q)\left(1^{2}+1 \cdot q+q^{2}\right)\left(1+q^{3}\right)}{1-q}$

$=a_{1}\left(1^{2}+1 \cdot q+q^{2}\right)\left(1+q^{3}\right)$

$=a_{1}\left(1^{2}+1 \times 1+1^{2}\right)\left(1+1^{3}\right)=a_{1}(1+1+1)(1+1)=6 a_{1}$

$S_{n}=n a_{1}$
上述求值方法非常类似高等数学当中求取极限的方法,

例如, 求 $\lim _{x \rightarrow 3} \frac{x-3}{x^{2}-9}$ 的 值: $\lim _{x \rightarrow 3} \frac{x-3}{x^{2}-9}=\lim _{x \rightarrow 3} \frac{x-3}{(x-3)(x+3)}$ $=\lim _{x \rightarrow 3} \frac{1}{(x+3)}=\frac{\lim _{x \rightarrow 3} 1}{\lim (x+3)}=\frac{1}{3+3}=\frac{1}{6}$ 。而实际上, 这类问题 往往又具有如午三个特征 : (1) 当 $x \rightarrow a$ 时, 无论分式的分 子还是分母都趋向于 0 ; (2)在 $x=x_{0}$ 的去心邻域内, 分子和 分母的导数都存在; ; (3) 分式在 $x=x_{0}$ 处的极限存在。这些 特征与洛必达法则的要求极为相似, 只是碍于分母非 0 的 传统，洛必达法则的要求更为苛刻而已。所以对于此类问 题，可以使用洛必达法则予以很好地解决，仍以上式为例： $\lim _{x \rightarrow 3} \frac{x-3}{x^{2}-9}=\lim _{x \rightarrow 3} \frac{(x-3)^{\prime}}{\left(x^{2}-9\right)^{\prime}}=\lim _{x \rightarrow 3} \frac{1-0}{2 x-0}=\lim _{x \rightarrow 3} \frac{1}{2 x}=\frac{1}{2 \times 3}=\frac{1}{6}$ 。

现在使用洛必达法则, 重新研究等比数列求和公式 的问题 : $\lim _{q \rightarrow 1} S_{n}=\lim _{q \rightarrow 1} \frac{a_{1}\left(1-q^{n}\right)}{1-q}=\lim _{q \rightarrow 1} \frac{\left[a_{1}\left(1-q^{n}\right)\right]^{\prime}}{(1-q)^{\prime}}=\lim _{q \rightarrow 1} \frac{a_{1}\left(0-n q^{n-1}\right)}{0-1}$ $=\lim _{q \rightarrow 1} n a_{1} q^{n-1}=n q_{1} \cdot 1^{n-1}=n a_{1}$ 。值得注意的是, $S_{n}=\frac{a_{1}-a_{n} q}{1-q}$ 却不适合上述做法。这是因为 $S_{n}=\frac{a_{1}\left(1-q^{n}\right)}{1-q}=\frac{a_{1}-a_{1} q^{m}}{1-q}$ $=\frac{a_{1}-\left(a_{1} q^{n-1}\right) q}{1-q}=\frac{a_{1}-a_{n} q}{1-q}$, 但是 $\frac{a_{1}-\left(a_{1} q^{n-1}\right) q}{1-q}=\frac{a_{1}-a_{n} q}{1-q}$ 对 于洛必达法则的使用发生了关于 $q$ 的“遮蔽”。

最后, 再来研究以 0 为项的常数列, 当 $a_{1}=a_{2}=\cdots=a_{n}=0$ 时, $q=\frac{a_{k+1}}{a_{k}}=\frac{0}{0}=1(0<k<n$ 且 $k \in N)$ ： $\lim _{a_{n} \rightarrow 0} S_{n}=n a_{1}=n \cdot 0=0$ ，求和公式同样适用。

9 对于一元二次函数 $a x^{2}+b x+c=0$ 当 $a=0, b \neq 0$ 时 , $0+b x+c=0 \Rightarrow b x=c \Rightarrow x=-\frac{c}{b}$ 。那么可否将 $a=0$ 直接代入求根公式 $x=\frac{-b \pm \sqrt{b^{2}-4 a c}}{2 a}$

$$
\begin{aligned}
& \text { 当 } b>0 \text { 时, } x_{1}=\frac{-b-\sqrt{b^{2}-4 a c}}{2 a}=\frac{-b-\sqrt{b^{2}-4 c \times 0}}{2 \times 0} \\
& =\frac{-b-\sqrt{b^{2}}}{0}=\frac{-b-b}{0}=\frac{-2 b}{0} \quad, x_{2}=\frac{-b+\sqrt{b^{2}-4 a c}}{2 a}=\lim _{a \rightarrow 0} \frac{\left(-b+\sqrt{b^{2}-4 a c}\right)^{\prime}}{(2 a)^{\prime}} \\
& =\lim _{a \rightarrow 0} \frac{\frac{-4 c}{2 \sqrt{b^{2}-4 a c}}}{2}=\lim _{a \rightarrow 0} \frac{-c}{\sqrt{b^{2}-4 a c}}=\frac{-c}{\sqrt{b^{2}-0}}=-\frac{c}{b} 。
\end{aligned}
$$

当 $b<0$ 时,$x_{1}=\frac{-b-\sqrt{b^{2}-4 a c}}{2 a}=\lim _{a \rightarrow 0} \frac{\left(-b-\sqrt{b^{2}-4 a c}\right)^{\prime}}{(2 a)^{\prime}}$ $=\lim _{a \rightarrow 0} \frac{-\frac{-4 c}{2 \sqrt{b^{2}-4 a c}}}{2}=\lim _{a \rightarrow 0} \frac{c}{\sqrt{b^{2}-4 a c}}=\frac{c}{\sqrt{b^{2}-0}}=-\frac{c}{b}, x_{2}=\frac{-b+\sqrt{b^{2}-4 a c}}{2 a}$ $=\frac{-b+\sqrt{b^{2}-4 c \times 0}}{2 \times 0}=\frac{-b+\sqrt{b^{2}}}{0}=\frac{-b-b}{0}=\frac{-2 b}{0}$ 。 
为什么总会多出一个 $\frac{-2 b}{0}$ 的根呢? 还是因为逻辑错 误。当 $a=0$ 时,$a x^{2}+b x+c=0 \Rightarrow a x^{2}+b x=-c \Rightarrow x^{2}+\frac{b}{a} x$ $=-\frac{c}{a} \Rightarrow x^{2}+\frac{b}{a} x+\left(\frac{b}{2 a}\right)^{2}=-\frac{c}{a}+\left(\frac{b}{2 a}\right)^{2} \Rightarrow\left(x+\frac{b}{2 a}\right)^{2}=\frac{b^{2}-4 a c}{4 a^{2}}$.

当 $b>0$ 时, $\left(x+\frac{b}{2 a}\right)^{2}=\frac{b^{2}-4 a c}{4 a^{2}} \Rightarrow x+\frac{b}{2 a}=+\sqrt{\frac{b^{2}-4 a c}{4 a^{2}}} \Rightarrow$ $x=-\frac{b}{2 a}+\sqrt{\frac{b^{2}-4 a c}{4 a^{2}}}=-\frac{b}{2 a}+\frac{\sqrt{b^{2}-4 a c}}{2 a}=\frac{-b+\sqrt{b^{2}-4 a c}}{2 a}$ $\lim _{a \rightarrow 0} \frac{\left(-b+\sqrt{b^{2}-4 a c}\right)^{\prime}}{(2 a)^{\prime}}=\lim _{a \rightarrow 0} \frac{\overline{2 \sqrt{b^{2}-4 a c}}}{2}=\lim _{a \rightarrow 0} \frac{-c}{\sqrt{b^{2}-4 a c}}=\frac{-c}{\sqrt{b^{2}-0}}=-\frac{c}{b}$

当 $b<0$ 时, $\left(x+\frac{b}{2 a}\right)^{2}=\frac{b^{2}-4 a c}{4 a^{2}} \Rightarrow x+\frac{b}{2 a}=-\sqrt{\frac{b^{2}-4 a c}{4 a^{2}}} \Rightarrow$ $x=-\frac{b}{2 a}-\sqrt{\frac{b^{2}-4 a c}{4 a^{2}}}=-\frac{b}{2 a}-\frac{\sqrt{b^{2}-4 a c}}{2 a}=\frac{-b-\sqrt{b^{2}-4 a c}}{2 a}$


值得注意的是, $\left(-\frac{b}{2 a}, \frac{4 a c-b^{2}}{4 a}\right)$ 同样不适合 $a=0$ 的情况, 因为此时并不存在对称轴 $x=-\frac{b}{2 a}$ 和顶点 $y=\frac{4 a c-b^{2}}{4 a}$, 所 以不能盲目代入 $a=0$ 。

\section{0 当 $x=0$ 时, 求函数 $y=\sin \frac{1}{x}$ 的值}

当 $x$ 从左右两侧不断趋近于 0 的时候, 函数 $y=\sin \frac{1}{x}$ 的 图像发生剧烈的振荡，因此习惯上称其为振荡函数， $x=0$ 称 为函数 $y=\sin \frac{1}{x}$ 的振荡间断点 (见图 6)。但是振荡间断点 既不是跳跃间断点，也不是无穷间断点。换句话说， $x=0$ 是在函数连续的情况下，由于缺失定义造成的可去间断点。 所以 $y=\sin \frac{1}{x}$ 作为奇函数, 当 $x=0$ 时, $y=\sin \frac{1}{0}=0$ 。

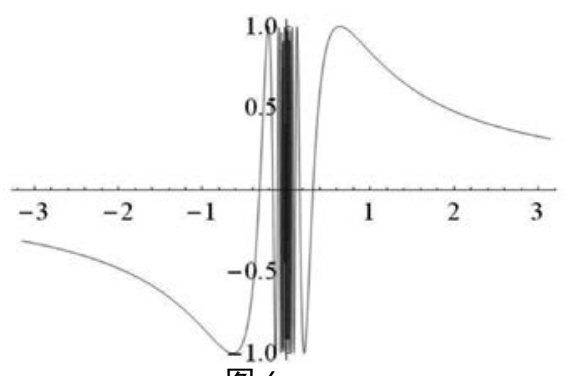

图 6

11 求正切函数 $y=\tan x$ 在 $x=\frac{\pi}{2}+k \pi, k \in Z$ 处 的值

正切函数 $y=\tan x$ 在 $x=\frac{\pi}{2}+k \pi, k \in Z$ 左右两侧的函
数值互为相反数, 并且分别趋向正负无穷, 所以习惯上称 $x=\frac{\pi}{2}+k \pi, k \in Z$ 为函数 $y=\tan x$ 的无穷间断点 (见图 7)。 那么引入“极数” 概念以后, 函数 $y=\tan x$ 在 $x=\frac{\pi}{2}+k \pi$, $k \in Z$ 处的函数值是多少呢? 对于这类问题, 便作规定如下, 当原间断点两侧的函数值不同，并且间断点处缺失定义的时 候，原间断点处的函数值以 $x$ 从左侧到达原间断点处的结果 为准。对于正切函数 $y=\tan x$, 将 $x$ 从左侧到达原间断点 $x=\frac{\pi}{2}+k \pi, k \in Z$ 的函数值 $\frac{1}{0}$, 作为该处的函数值。即当 $x=\frac{\pi}{2}+k \pi, k \in Z$ 时, $y=\tan x=\frac{1}{0}$ 。

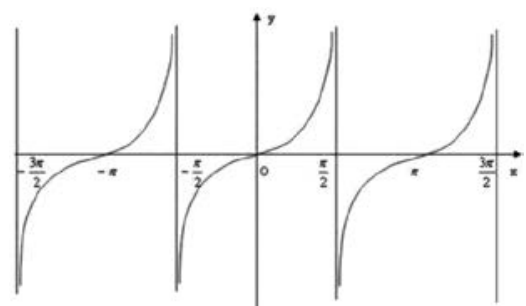

图 7

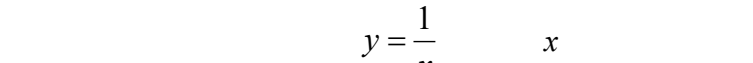
限趋近于 0 时, 两侧的函数值互为相反数, 并且不断趋向正 负无穷, 这意味着 $x=0$ 为函数的无穷间断点 (见图 8)。当 $x$ 从左侧到达 0 处时的函数值为 $-\frac{1}{0}$, 便将 $-\frac{1}{0}$ 作为函数在 $x=0$ 处的值。即当 $x=0$ 时, $y=-\frac{1}{0}$ 。



图 8

12 在条件概率 $P(B \mid A)=\frac{P(A B)}{P(A)}$ 中, $P(A)$ 是否可以为 0

对于条件概率 $P(B \mid A)=\frac{P(A B)}{P(A)}$, 当 $P(A)=0$ 时依然适用。例如, 在 5 道题中有 3 道理科题和 2 道文 科题。如果不放回地依次抽取 2 道题，设“第 1 次抽到 
文理综合题”为事件 $A$ “" 第 2 次抽到理科题” 为事件 $B$, 则“第 1 次和第 2 次都抽到理科题” 就是事件 $A B^{[3]}$, 即有 $P(B \mid A)=\frac{P(A B)}{P(A)}=\frac{P(A) \cdot P(B)}{P(A)}=\lim _{P(A) \rightarrow 0} \frac{[P(A) \cdot P(B)]^{\prime}}{[P(A)]^{\prime}}=\frac{P(B)}{1}$ $=P(B)=\frac{3}{5} \times \frac{2}{4}+\frac{2}{5} \times \frac{3}{4}=\frac{3 \times 2+2 \times 3}{5 \times 4}=\frac{6+6}{20}=\frac{12}{20}=\frac{3}{5}$ 。

13 当 $\sigma=0$ 时, 正态分布函数 $f(x)=\frac{1}{\sqrt{2 \pi} \sigma} e^{-\frac{(x-\mu)^{2}}{2 \sigma^{2}}}$, $x \in(-\infty,+\infty)$ 的图像面积是否为 0

在正态分布函数 $f(x)=\frac{1}{\sqrt{2 \pi} \sigma} e^{-\frac{(x-\mu)^{2}}{2 \sigma^{2}}}, \mathrm{x} \in(-\infty,+\infty)$ 中 , $\mu$ 、 $\sigma$ 分别表示总体的平均数和标准差。曲线关于直线 $x=\mu$ 对称, 且在 $x=\mu$ 处位于最高点。当 $\mu$ 一定时，曲线的形状由 $\sigma$ 确定。 $\sigma$ 越大，曲线越“矮胖”，表示总体的分布越分散 $; \sigma$ 越小， 曲线越“瘦高”，表示总体的分布越集中；当 $\sigma$ 相同时，曲 线的位置由 $\mu$ 决定。曲线在 $x$ 轴的上方, 与 $x$ 轴所围面积为 1 (见图 9)。



图 9

当 $\sigma=0$ 时, $x=\mu=0$, 代 入上式 $f(\mu)=\frac{1}{\sqrt{2 \pi} \cdot 0} e^{-\frac{(\mu-\mu)^{2}}{2 \times 0^{2}}}$ $=\frac{1}{0} \cdot e^{-\frac{0^{2}}{2 \times 0^{2}}}=\frac{1}{0} \cdot e^{-\frac{0^{2}}{0^{2}}}=\frac{1}{0} \cdot e^{\frac{0}{0}}=\frac{1}{0} \cdot e^{1}=\frac{1}{0}$, 此时曲线与 $x$ 轴所 围面积 : $S=0 \times \frac{1}{0}=1$ 。

14 函数 $y=0 \cdot x$ 与直线 $y=0$ 的图像一样吗

通常来讲, 0 乘以任何数都等于 0 。但是现在引入

了“极数” 概念, 并且 $0 \times \frac{1}{0}=1$ 。当 $x_{1}=\frac{1}{2} \times \frac{1}{0} 、 x_{2}=\frac{1}{3} \times \frac{1}{0}$ 、 $x_{3}=\frac{1}{4} \times \frac{1}{0} 、 \cdots \cdots$ 的时候, $y$ 又等于多少呢? 将这些数值 分别代入上式: $y_{1}=0 \times \frac{1}{2} \times \frac{1}{0}=\frac{1}{2}, y_{2}=0 \times \frac{1}{3} \times \frac{1}{0}=\frac{1}{3}$, $y_{3}=0 \times \frac{1}{3} \times \frac{1}{0}=\frac{1}{3}, \cdots \cdots$ 。由此可知, 当 $x=\infty$ 时, 因为 $\infty$ 可以看做与 $\frac{1}{0}$ 同等量级的数, 即 $\infty=a \cdot \frac{1}{0} \quad(0<a<1)$, 所 以 $y=0 \times \infty=0 \cdot a \cdot \frac{1}{0}=a$, 而非 $y=0 \times \infty=0$ 。可以看到， 引入“极数” 概念以后, 原本的某些运算法则开始出现不适。 这就很像物理学中引入相对论后, 低速世界的运动规律不再
适用高速世界一样。

15 幂函数 $y=x^{0}$ 与直线 $y=1$ 的图像是否一样 对于幂函数 $y=x^{0}$,总有 $\cdots=(-3)^{0}=(-2)^{0}=(-1)^{0}=1=(1)^{0}$ $=(2)^{0}=(3)^{0}=\cdots$ 。除 $x=0$ 以外, 各点所对应的函数值均 为 1 。当 $x=0$ 时, $y=x^{0}=1$ 。因此函数的图像是一条与 $y$ 轴交 于 $(0,1)$, 连续不断的水平直线, 它与直线 $y=1$ 的图像一样。

\section{6 函数 $y=0^{x}$ 的图像如何}

当 $x>0$ 时, $y=0^{x}=0$; 当 $x=0$ 时, $y=0^{x}=0^{0}=1$; 当 $x<0$ 时, $y=0^{x}=0^{-(-x)}=0^{-1 \cdot(-x)}=\left(0^{-1}\right)^{-x}=\left(\frac{1}{0}\right)^{-x}$ 。由上可知 , $x=0$ 是 函数 $y=0^{x}$ 的跳跃间断点。当 $x<0$ 时, $y=\left(\frac{1}{0}\right)^{-x}$; 当 $x=0$ 时, $y=1$; 当 $x>0$ 时, $y=0$ 。

\section{7 做出函数 $y=\tan x \cdot \cos x$ 的图像}

当 $x \neq \frac{\pi}{2}+k \pi, k \in Z$ 时, 则 $y=\tan x \cdot \cos x=\frac{\sin x}{\cos x} \cdot \cos x$ $=\sin x$ 。当 $x=\frac{\pi}{2}+k \pi, k \in Z$ 时, 函数就会出现间断点。 现在引入“极数” 概念, : 当 $x=\frac{\pi}{2}+k \pi, k \in Z$ 时, 函数 $y=\tan x \cdot \cos x=\frac{\sin x}{\cos x} \cdot \cos x=\frac{\sin x \cdot \cos x}{\cos x}=\lim _{\cos x \rightarrow 0} \frac{(\sin x \cdot \cos x)^{\prime}}{(\cos x)^{\prime}}=\frac{\sin x}{1}=\sin x= \pm 1$ (依据实际情况, 判断 $y=1$ 或者 $y=-1$ )。该值恰 好等于间断点左右邻域的函数极限，便将 $y= \pm 1$ (同上) 作为函数在各间断点处的值。如此一来, $y=\tan x \cdot \cos x=\frac{\sin x}{\cos x} \cdot \cos x=\sin x, x \in R$, 不再担心 $x=\frac{\pi}{2}+k \pi$, $k \in Z$ 时产生间断点了 (见图 10)。

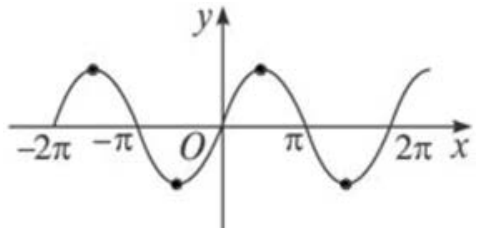

图 10

\section{8 “极数” 的特殊性质}

根据定义, 发现“极数” 很像物理学中的光速, 具有 一些普通数字所不具有的特殊性质：

第一, (1) $\frac{1}{0}$ 就像 $\infty$ 一样具有正负的区别, $\frac{1}{0}-\frac{1}{0}=0$ : $+\frac{1}{0}$ 类似 $+\infty$ 表示正向的极数,$+\frac{1}{0}=\frac{1}{0}$ 。 $-\frac{1}{0}$ 类似 $-\infty$ 表 示负向的极数, $\frac{1}{0}$ 前面的“ 
是 0,0 的倒数是 $\frac{1}{0}$; (3) $\frac{1}{0}$ 所代表的是一个确定的数值, 而 非 $\infty$ 那样代表一个笼统的概念，即 $\pm \infty \pm a= \pm \infty$ 。因此 二维坐标系实际上是一个边长为 $\frac{1}{0}$ 的正方形平面, 原点距 离各边的长度为 $\frac{1}{2} \times \frac{1}{0}$ 。三维坐标系实际上是一个边长为 $\frac{1}{0}$ 的立方几何体, 原点距离各面的长度为 $\frac{1}{2} \times \frac{1}{0}$ 。

第二，关于 $\frac{1}{0}$ 的四则运算 :

(1) $\frac{1}{0}+a=\left\{\begin{array}{l}t, \text { 且 } 0 \leq t<\frac{1}{0}(a<0) \\ \frac{1}{0}(a=0) \\ \frac{1}{0}(a>0)\end{array}\right.$

(2) $\frac{a}{0}=\left\{\begin{array}{l}\frac{1}{0}(a \geq 1) \\ t, \text { 且 } 1<t<\frac{1}{0}(0<a<1) \\ 1(a=0) \\ t, \text { 且 }-\frac{1}{0}<t<0(-1<a<0) \\ -\frac{1}{0}(a \leq-1)\end{array}\right.$

$$
\text { (3) } \left.\begin{array}{c}
\frac{a \cdot 0}{b \cdot 0} \\
\frac{a \cdot 0}{b \cdot 0}
\end{array}\right\}=\frac{0}{0}=1
$$

$(a, b \in r, r$ 为原 $R, R$ 为引入“完全定理” 以后的全 体实数)

第三, 0 与非 0 的运算先于 0 与 0 的运算。例如，按照 比例的基本性质, 如果 $\frac{a}{b}=\frac{c}{d} \Rightarrow \frac{d}{b}=\frac{c}{a}$ ，那么 $\frac{0}{b}=\frac{0}{d} \Rightarrow \frac{d}{b}=\frac{0}{0}$ 。
显然这是错的, 该式应为 $\frac{0}{b}=\frac{0}{d} \Rightarrow \frac{0}{1}=\frac{0}{1} \Rightarrow 0=0$ 。 又如, $\frac{1}{0}=\frac{2}{0}=\frac{3}{0}=\cdots$, 虽然式中分母相同, 但是不能由此得出 $1=2=3=\cdots$ 的结论，应将分子、分母同时约去分子本身。可 以看到, 无论 0 做分子还是分母, 0 与非 0 的运算先于 0 与 0 的运算。

第四，使用极限方法求值或者等号两边同时除以 0 时， 必须注意由于数字整合导致的运算“遮蔽”。例如, 当 $q=1$ 时， $\frac{a_{1}-\left(a_{1} q^{n-1}\right) q}{1-q}=\frac{a_{1}-a_{n} q}{1-q}$ 就对 $q$ 的运算发生了“遮蔽”。 又如, 当 $m=n$ 时, $a_{n}-a_{m}-0=(n-m) d \Rightarrow \frac{a_{n}-a_{m}-0}{n-m}=d$ 就对 $n=m$ 的运算发生了遮蔽。

第五, 0 做分母时, 方程具有不可逆性，即无法还原得 到原方程式。例如, $y=k x(k \geq 1) \Rightarrow \frac{y}{0}=\frac{k x}{0} \Rightarrow \frac{k x}{0}-\frac{y}{0}$ $=0 \Rightarrow \frac{x}{0}-\frac{y}{0}=0 \Rightarrow x-y=0 \Rightarrow y=x$ 。显然, 直线 $y=x$ 只是直线 $y=k x(k \geq 1)$ 中的一条。

\section{9 结语}

总而言之 “完全定理”的引入非常必要。它既解决了 一系列的、长期困扰数学领域的问题, 又填补了许多一直被 人忽视的研究空白。“极数”的特殊性质及其运算法则更为 数学的研究带来了全新的认识和重要的启示, 并将数学的研 究推进到了更为深远的领域。

\section{参考文献}

[1] 课程教材研究所. 数学必修 4 [M]. 北京 : 人民教育出版社, 2007.

[2] 同济大学数学系. 高等数学 [M]. 第六版. 北京 : 高等教育出版 社 , 2007.

[3] 课程教材研究所. 数学选修 2-3[M]. 北京: 人民教育出版 社 ,2009. 